\title{
Mitochondrial dysfunction and mitophagy in Parkinson's disease: from mechanism to
} therapy

3 Ana Belen Malpartida ${ }^{1}$, Matthew Williamson ${ }^{1}$, Derek P Narendra ${ }^{2}$, Richard Wade-Martins*1 4 and Brent J Ryan*1

1. Oxford Parkinson's Disease Centre, Department of Physiology, Anatomy and Genetics, Le 7 Gros Clark Building, University of Oxford, South Parks Road, Oxford, OX1 3QX.

8 2. Inherited Movement Disorders Unit, National Institute of Neurological Disorders and 9 Stroke, Bethesda, MD.

*To whom correspondence should be addressed

12 Brent.ryan@dpag.ox.ac.uk or Richard.wade-martins@dpag.ox.ac.uk

\section{Key words}

15 Neurodegeneration, Bioenergetics, $\alpha$-synuclein, PINK1, Parkin, Deubiquitylase

\section{Abstract}

Mitochondrial dysfunction has been associated with neurodegeneration in Parkinson's disease (PD) for over 30 years. Despite this, the role of mitochondrial dysfunction as an initiator, propagator, or bystander remains undetermined. The discovery of the role of the PD familial genes PINK1 and PRKN (parkin) in mediating mitochondrial degradation (mitophagy) reaffirmed the importance of this process in PD aetiology. Recently, progress has been made in understanding the upstream and downstream regulators of canonical PINK1/parkinmediated mitophagy, alongside non-canonical PINK1/parkin mitophagy, in response to mitochondrial damage. Progress has also been made in understanding the role of PDassociated genes such as SNCA, LRRK2, and CHCHD2 in mitochondrial dysfunction and their overlap with sporadic disease, opening up opportunities for therapeutically targeting mitochondria in PD. 
Parkinson's disease (PD) (see Glossary) is a common neurodegenerative disorder characterised by loss of dopaminergic neurons in the substantia nigra pars compacta (SNpc). This results in the loss of dopaminergic inputs in the striatum and the characteristic motor symptoms associated with the disease. Accumulation of aggregated $\boldsymbol{\alpha}$-synuclein in Lewy bodies are the pathological hallmark of PD in the SNpc, but can also be observed in other affected brain regions.

Mitochondrial dysfunction has been recognised as an important initiating factor in dopaminergic neuronal loss for over 30 years given that toxins inhibiting complex I induce dopaminergic cell loss and parkinsonism, complex I is dysfunctional in sporadic PD patient tissues, and the familial autosomal recessive PD genes PINK1 and PRKN (parkin) have been discovered to play a key role in mitochondrial quality control.

This review explores the recent progress in understanding the regulation of the PINK1/parkin pathway in PD pathogenesis, the roles of established PD genes such as SNCA and LRRK2 in mitochondrial function, and the discovery of new PD-associated genes with mitochondrial roles, such as $\mathbf{C H C H D 2}$. Additionally, we discuss recent data highlighting commonalities in mitochondrial dysfunction in sporadic disease and monogenic PD, in addition to presenting the therapeutic opportunities presented by recognition of mitochondrial dysfunction and impaired mitophagy in PD.

\section{Regulation of the PINK1/parkin pathway and its role in mitophagy} Mutations in the PINK1 (PARK6) and parkin (PARK2) genes were among the first to be linked to autosomal recessive early-onset PD [1, 2]. Although mutations in both genes presented with similar clinical phenotypes, their contribution to PD pathology remained undefined until the role of PINK1 and parkin in mitophagy was described in 2010 [3].

The mechanism of PINK1/parkin activation has been thoroughly investigated and reviewed in detail [4]. However, recent advances have added further to our knowledge of both inducers and inhibitors of this pathway (Box 1). These new findings have implications for PD pathophysiology and therapeutic developments for PD patients both with and without PINK1 or PRKN mutations.

63

Structural information provides insights into the mechanism of PINK1/parkin activation The most up-to-date model for parkin activation by PINK1 suggests that following mitochondrial membrane depolarization, PINK1 is stabilized at the outer mitochondrial membrane (OMM) where it phosphorylates pre-existing ubiquitin (Ub) at serine 65 residues (pSer65Ub) [5]. Auto-inhibited cytosolic parkin is then recruited to sites of PINK1 activity where RING1 of parkin domain binds pSer65Ub initiating a series of conformational changes 
[6]. Notably, parkin remains in a partially auto-inhibited form once bound to pSer65Ub and is only fully activated by phosphorylation of its Ub-like domain by PINK1 [7] (Figure 1).

The role of phosphorylation of ubiquitin at serine 65 is increasingly appreciated as a marker of PINK1-induced pathway activation, leading to the recruitment of parkin in a feedforward mechanism (Figure 1). Once active, parkin synthesises Ub chains on OMM proteins, providing more substrates for PINK1 ubiquitin phosphorylation, which in turns recruits more parkin, ultimately leading to maximally active ( $\sim 4,400$-fold activation) Ub chain assembly (as reviewed in [8]). Evidence of increased pSer65Ub formation has been observed in postmortem PD brain and diminished in patients with PINK1/PRKN mutations, indicating the relevance of this pathway in disease $[9,10]$.

Although a structure of fully active parkin has not yet been reported, the structure of PINK1, alone and in complex with $\mathrm{Ub}$, has been thoroughly investigated. It has been shown that PINK1 preferentially binds to a Ub conformation with a retracted C-terminus (Ub-CR) at a 50fold higher rate than the wildtype Ub conformation [11]. The Ub-CR conformation has an extended Ser65 loop that promotes its binding to the kinase-activation segment of PINK1 whereas non-phosphorylated $\mathrm{Ub}$ is unable to bind to this domain directly. However, it has been suggested that unmodified Ub can bind to PINK1 and be transformed to the Ub-CR conformation while bound, stabilizing the complex and promoting subsequent phosphorylation of Ser65 residues [11].

Regulators of PINK1 and parkin

93 Regulators of PINK1 and parkin protein levels have been recently identified, offering mechanistic and therapeutic insights. For example, genome-wide CRISPR screens have been used to identify a transcriptional repressor network, including THAP11, which negatively regulates parkin levels and mitophagy [12], and the adenine nucleotide translocator (ANT) complex which regulates TIM23-mediated translocation and stabilization of PINK1, upstream of depolarisation-induced mitophagy [13]. Similarly, Gas7 is another regulator found to act upstream of PINK1, regulating PINK1 protein levels and pSer65Ub formation, with the loss of Gas7 impairing mitophagic flux [14].

Post-translational regulation of both PINK1 and parkin have been demonstrated as additional mechanisms to regulate their activity. Several kinases, including AKT, have been proposed to initiate PINK1/parkin dependent mitophagy in various cell models, including induced pluripotent stem cell (iPSC)-derived dopaminergic neurons [15], linking upstream signalling pathways in cell stress to the initiation of mitophagy. Both ageing and $\alpha$-synuclein oligomers have been demonstrated to increase parkin S-nitrosylation, increasing parkin autoubiquitination and subsequent degradation [16, 17], highlighting S-nitrosylation as an important regulator of mitophagy. Excessive PINK1 S-nitrosylation can impair PINK1 kinase 
110 activity resulting in increased neuronal death in both iPSC-derived dopaminergic neurons and

$111 \alpha$-synuclein transgenic PD mice $[18,19]$.

112

\section{Deubiquitylases as gatekeepers of mitophagy}

114 A number of deubiquitylases (DUBs), including USP8, USP14, USP15, USP35, and most 115 prominently USP30, have been found to regulate mitophagy by antagonising parkin activity, 116 acting either directly on parkin or its substrates $[8,20]$.

118 USP30 is an established OMM-localised enzyme which negatively regulates PINK1/parkin119 dependent mitophagy through deubiquitylation of several OMM substrates including RHOTs, 120 VDACs, MFNs, and components of the OMM translocon [21, 22] (Figure 1). In vitro studies 121 have demonstrated that USP30 shows selectivity for cleavage of K6- and K11-linked Ub chains $122[21,23]$ with increased binding to K6-linked Ub chains, and increased hydrolysis of K11-linked 123 Ub chain stoichiometry. USP30 demonstrates a lower affinity for cleavage of pSer65Ub chains 124 allowing the synthesis of phosphorylated ubiquitin chains during PINK1/parkin activation [17, $12523,24]$. However, the role of USP30 in maintaining mitochondrial quality control in

128 Recent studies have also established a role for USP30 in regulating mitophagy under basal 129 conditions independently of PINK1/parkin activity (Box 2) [22, 25]. Interestingly, knockdown 130 of PINK1 (but not parkin) was able to suppress the enhancement of mitophagy seen upon 131 USP30 knockdown, suggesting that USP30 acts upstream of PINK1 to set the threshold for 132 initiating mitophagy $[22,25,26]$. Furthermore, two classes of ubiquitylation events were 133 identified to be more abundant in USP30 depleted iPSC-derived induced neurons [27] being:

134 (1) OMM protein ubiquitylation was elevated under basal conditions but not during 135 depolarisation (e.g TOM40 and VDAC2); and (2) OMM protein ubiquitylation was elevated 136 under basal conditions and further increased during depolarisation (e.g TOM20, TOM5 and 137 VDAC1). Interestingly, these classes of substrates may represent targets of PINK1138 independent and PINK1-dependent mitophagy, respectively, giving an insight into the role of 139 USP30 in regulating mitochondrial homeostasis and mitophagy.

Under basal conditions, USP30 knockout (KO) neurons display increased ubiquitylation of proteins normally imported into the mitochondrial matrix or inner membrane via the TOM complex $[27,28]$. Constitutive ubiquitylation during import of mitochondrial proteins relies on the E3 Ub ligase March5 and is antagonised by USP30, thereby dynamically regulating mitochondrial protein import [28]. However, March5 knockdown did not fully eliminate intramitochondrial protein ubiquitination, suggesting that additional E3 Ub ligases function upstream of USP30 [28].

USP30 has a multi-faceted role in mitochondrial quality control, making it an attractive target for therapeutic intervention in diseases characterised by mitochondrial dysfunction. Further 
151 elucidation of the interplay between USP30, PINK1, parkin and other E3 Ub ligases in complex

152 neuronal models, especially in the context of PD, are needed.

Identification of pSer65Ub phosphatases as negative regulators of mitophagy

155 Ub phosphorylated at Ser65 by PINK1 undergoes structural changes to become resistant to 156 DUB activity [29]. Dephosphorylation of pSer65Ub is, therefore, a potentially critical negative regulatory mechanism in the modulation of mitophagy (Figure 1).

Recently, phosphatases such as PTEN-Long (PTEN-L or PTEN- $\alpha$ ) and PPEF2 have been demonstrated to regulate pSer65Ub phosphorylation and act as negative regulators of mitophagy [30, 31]. These phosphatases have been demonstrated to localise to the OMM and maintain mitochondrial quality control in both neurons and astrocytes in vitro and in vivo [32,

163 33]. In addition, recent reports demonstrated that PINK1 phosphorylates Ub in astrocytes to 164 a greater extent than in neurons [34]. Although further studies are needed, this raises the 165 possibility that phosphorylation and dephosphorylation of mitochondrial proteins may play a role in modulating mitochondrial dysfunction primarily in non-neuronal cells.

PTEN-L is a translational variant of PTEN and was the first specific phosphatase described to antagonize PINK1 function by acting on pSer65Ub chains. PTEN-L knockdown significantly increasing Ub phosphorylation at Ser65 in a parkin-dependent manner [30]. More recently, PPEF2 was identified as another phosphatase antagonizing PINK1 activity [33]. Like PTEN-L, PPEF2 overexpression was shown to significantly reduce the rate of pSer65Ub formation in cortical neuronal cultures. However, in parkin-overexpressing models the effect of PPEF2 was significantly diminished [33] as the strength of the PINK1/parkin feedforward mechanism appeared to outweigh the rate of dephosphorylation. Additionally, PTEN-L KO mice displayed a decrease in mitophagy shown by impaired parkin recruitment in cardiomyocytes [32], suggesting that increased phosphorylation of $\mathrm{Ub}$ is not the sole determinant for increasing mitophagy.

Together, these recent advances in understanding the mechanisms which promote and restrain PINK1/parkin mitophagy have provided a clearer picture of mitochondrial homeostasis and canonical PINK1/parkin mitophagy. This information allows an understanding of the likely phenotypic consequences of mutations in different regions of PINK1 or parkin for PD patients, as well as understanding the potential therapeutic opportunities to enhance PINK1/parkin mitophagy by increasing expression levels or activity.

187 Loss-of-function mutations in either PINK1 or parkin are associated with the development of 188 autosomal recessive familial PD in humans. It is therefore important to understand the role 189 of PINK1 and parkin in PD beyond canonical PINK1/parkin-dependent mitophagy (Box 2). Early 190 Pink1/Prkn KO mouse models failed to recapitulate PD pathology, and mitochondrial defects 
191 were only found in midbrain-specific KO models [35]. However, locomotor deficits and

192 dopaminergic neuron loss have recently been observed in two-year-old Prkn KO mice [36].

193 These observations suggest a subtle role for mitophagy in age-dependent neurodegeneration

194 and further emphasise the preferential vulnerability of dopaminergic neurons (Box 3).

196 Isogenic PRKN KO iPSC-derived dopaminergic neurons have been demonstrated to have 197 substantial changes in both their native and post-translational proteome, resulting in 198 disruption of both mitochondrial and non-mitochondrial phenotypes including impaired RhoA 199 signalling which results in impaired neurite outgrowth [37]. In addition, loss of PRKN 200 decreases the activity of lysosomal enzymes, including GBA (GCase). Given that parkin also 201 targets several vacuolar ATPases for degradation [38], together these data suggest a loss of 202 PRKN has an impact downstream of mitochondria [39] affecting cellular processes beyond 203 mitochondrial homeostasis. iPSC-derived dopaminergic neurons from PD patients with 204 compound heterozygous PRKN mutations demonstrate a loss of parkin and an inability to 205 release ER-mitochondrial contact sites in response to mitochondrial depolarisation [40]. 206 Additionally, a comparison of several monogenic PD rat models using in vivo microdialysis has 207 demonstrated alterations in dopamine and/or dopamine metabolites as well as alterations in 208 other neurotransmitters in PINK1 and parkin KO rats [41].

210 Furthermore, PINK1/parkin modulates inflammation in response to activation of the STING 211 pathway induced by mitochondrial DNA damage [42]. Loss of Pink1 has also been shown to 212 increase mitochondrial antigen presentation (MiTAP) with bacterial challenge of Pink1 KO 213 mice demonstrating cytotoxic (CD8+) T-cell responses in both the gut and brain [43]. Given 214 that MITAP induction correlated with loss of dopaminergic neuronal axonal varicosities in the 215 striatum [43], the role of MITAP and peripheral inflammation in PD and particularly 216 PINK1/parkin mutation patients is of great interest.

\section{The effects of mutations in PD causing genes on mitochondrial function and mitophagy}

Recent findings have greatly increased our understanding of the mechanisms by which established monogenic PD-associated proteins alter mitochondrial function and mitophagy, providing interesting insights into overlap between mechanisms involved in monogenic PD. In addition to the long-established autosomal dominant familial mutations in SNCA and $\angle R R K 2$, other genes implicated in mitochondrial function/dysfunction carry inherited PD mutations, such as CHCHD2 (PARK22), GCH1, and VPS35 (PARK17), and, in some cases, also show association with PD in genome-wide association studies (GWAS). Understanding how these pathways trigger nigral degeneration will inform on mechanisms in both familial and sporadic PD. 
a-synuclein, the main component of Lewy bodies, has long been associated with disruption of mitochondrial function but without a clear mechanistic rationale. In recent years, significant developments have been made in assessing the mitochondrial targets affected by $\alpha$-synuclein and the $\alpha$-synuclein species responsible for these effects (Figure 2 ). For instance, overexpression of $\alpha$-synuclein or the addition of exogenous aggregated $\alpha$-synuclein species has shown $\alpha$-synuclein to interact with several OMM components including TOM20 [44, 45], VDAC [46], and $F_{1} F_{0}-A T P$ synthase, causing mitochondrial permeability transition pore (MPTP) opening [47]. Accumulated pathological $\alpha$-synuclein, or dopamine-modified or phosphomimetic species mimicking pathological forms of the protein, have been shown to preferentially bind to mitochondria [44,48], inhibiting mitochondrial protein import and leading to mitochondrial membrane depolarization and impaired cellular respiration [48].

Models of seeded $\alpha$-synuclein aggregation demonstrated decreased levels of MFN-2 and OPA1 levels [49], consistent with previous reports of $\alpha$-synuclein-induced mitochondrial fragmentation and bioenergetic alterations in iPSC-derived dopaminergic neurons [45]. Proteomic analyses revealed significant recruitment of proteins involved in oxidative phosphorylation into aggregates consistent with impairments in respiration. Furthermore, recent studies have demonstrated that the process of Lewy body formation, rather than aggregation itself, is the key driver for dysfunction induced by $\alpha$-synuclein [49] Mitochondrial dysfunction and complex I deficits are temporally separated from pS129 formation induced by $\alpha$-synuclein fibrils in hippocampal neurons, suggesting additional mechanisms beyond the interaction between $\alpha$-synuclein and the mitochondria are needed for dysfunction [49]. Indeed, Lewy bodies from PD patients have been found to contain fragmented mitochondria crowded with lipids and lysosomes as well as $\alpha$-synuclein [50], again suggesting that mitochondrial dysfunction, $\alpha$-synuclein aggregation and Lewy body formation are intrinsically linked.

261 A growing body of evidence links LRRK2 mutations to PD-associated mitochondrial dysfunction (Figure 3). It has been shown in iPSC-derived dopamine neurons that LRRK2 can regulate mitophagy by removing the OMM adaptor protein MIRO from the MIRO/MILTON/KINESIN motor complex resulting in reduced mitochondrial transport along the cytoskeleton, reducing engulfment by autophagosomes and subsequent degradation [51]. The pathogenic G2019S mutation in the kinase domain of LRRK2 disrupts this function, slowing the initiation of mitophagy [51]. These observations are in agreement with previous reports of impaired mitochondrial trafficking in LRRK2-R1441C mutant rat neurons [52]. Importantly, these phenotypes have also been observed in neuronal cultures from idiopathic 

in the pathology of PD [51].

273 Furthermore, two independent studies have reported that mutant LRRK2 can impair 274 PINK1/parkin-dependent mitophagy via independent mechanisms $[53,54]$. The increased 275 kinase activity of the LRRK2-G2019S mutant was shown to disrupt protein-protein 276 interactions on the OMM early in PINK1/parkin-dependent mitophagy, including the 277 recruitment of the mitochondrial fission protein Drp1 and parkin [53]. In addition, both the 278 G2019S and R1441C mutations in LRRK2 impair later stages of PINK1/parkin-dependent 279 mitophagy through increased phosphorylation of the small GTPase RAB10 inhibiting its 280 interaction with the autophagy receptor OPTN and reducing accumulation on depolarised mitochondria [54]. However, given the differential expression of LRRK2 between brain regions and cell types [55], the role of LRRK2 in initiating mitochondrial dysfunction solely in neurons cannot be assumed.

Further advances have identified mitochondrial roles for additional PD-associated genes. Whilst many of the details of the mechanisms of these dysfunctions remain to be elucidated, including the precise effects of these mutations on mitochondrial turnover, the convergence of these dysfunctions are of great interest to improve our understanding of PD pathology. Heterozygous mutations in GBA, which encodes the lysosomal enzyme GCase, responsible for degrading glucosylceramide, are the strongest genetic risk factors for PD. Recently, analysis of post-mortem brain tissue from PD patients carrying heterozygous GBA mutations revealed increased mitochondrial content, increased mitochondrial oxidative stress, and impaired autophagy [56]. These findings mirror previous observations in iPSC-derived dopaminergic neuronal cultures from patients with GBA-L444P mutations and GBA KO neurons [57]. Furthermore, primary hippocampal neurons from $G b a^{L 444 P / W T}$ knock-in mice displayed abnormal mitochondrial morphology, increased mitochondrial oxidative stress, and defects in basal and PINK1/parkin-dependent mitophagy [56], again pointing toward a more general defect in mitophagy in the pathology of PD.

CHCHD2

303 Mutations in the CHCHD2 gene were identified as a novel familial PD gene in 2015 [58]. 304 CHCHD2 encodes a protein which modulates mitochondrial function in conjunction with the 305 ALS/FTD-associated gene CHCHD10 [59]. Further analysis of this pathway has identified that 306 CHCHD2 accumulates in damaged mitochondria and regulates CHCHD10 oligomerisation [60].

308 CHCHD2 has been shown to play a significant role in the maintenance of mitochondrial cristae 309 [59] as well as stabilising OPA1 to promote mitochondrial fusion [61]. Mutations in CHCHD2 310 have been demonstrated to induce precipitation of both mutant and wild type (WT) protein 
311 in the intermembrane space (IMS) [61] as well as cytochrome $\mathrm{C}$ destabilization, impaired

312 respiration, and mitochondrial ROS generation [62]. Moreover, CHCHD2 mutations have been

313 associated with $\alpha$-synuclein aggregation and oligomerization in human post-mortem brain

314 tissues and iPSC-derived dopaminergic neurons [63]. Although CHCHD2 has been

315 demonstrated to play a vital role in mitochondrial health, its potential role in the induction of

316 mitophagy remains to be elucidated.

317

$318 \quad$ GCH1

319 GTP-cyclohydrolase $1(\mathrm{GCH} 1)$ is the rate-limiting enzyme in synthesis of tetrahydrobiopterin 320 (BH4), a cofactor for enzymes including tyrosine hydroxylase and nitric oxide synthase, as well 321 as acting as an antioxidant. Polymorphisms in the GCH1 gene are enriched in PD patients and rare mutations have been identified in up to $0.75 \%$ of PD patients [64]. $\alpha$-synuclein has also been demonstrated to alter $\mathrm{GCH} 1$ activity [65]. In addition to the cytoplasmic role of $\mathrm{BH} 4$, it has been demonstrated that $\mathrm{BH} 4$ plays a role in mitochondrial function and redox regulation [66]. The absence of $\mathrm{BH} 4$ results in decreased mitochondrial function and increased levels of DRP1 activation, proteome remodelling and metabolic alterations [66].

327

VPS35 plays a role in recycling DLP1 complexes, with mutations and oxidative stress increasing VPS35 interactions and mitochondrial fission [67]. Parkin has been shown to poly-ubiquitinate VPS35 affecting endosomal sorting WASH complex-mediated retromer [38]. More generally, VPS35 mutations disrupt autophagosome formation through ATG9a, inhibiting autophagosome formation and vesicle transport between mitochondria and peroxisomes [68]. In addition, inhibition of retromer function results in accumulation of hyperactive (GTPbound) RAB7 on lysosomes inhibiting ATG9a and autophagosome formation [69]. Given the established link between LRRK2 and VPS35 [70], the similarity between mitochondrial phenotypes in VPS35 and LRRK2 models is noteworthy.

\section{Relevance to sporadic disease}

341 As only $\sim 15 \%$ of PD can be attributed to a monogenic cause, research into the molecular

342 mechanisms of sporadic PD (SPD) is vital. A number of recent studies have investigated 343 sporadic disease, identifying overlap between sporadic and monogenic phenotypes which 344 may lead to a greater understanding of the interplay between dopaminergic neuronal physiology and genetics in PD.

347 Increased pSer65Ub has been observed in post-mortem PD brain and neuronal models of PD $348[9,10,71]$ but decreased in PINK1/PRKN mutation carriers, demonstrating the relevance of 349 mitochondrial damage and mitophagy induction via the PINK1/parkin pathway in PD patients. 350 A comprehensive study of pSer65Ub in post-mortem brain from Lewy Body Disease demonstrated a significant correlation between pSer65Ub and Braak tau tangle staging in the 
hippocampus and with Lewy bodies in the amygdala, but not the substantia nigra [9]. However, how these observations apply to the general SPD population and the upstream- or downstream role of pSer65Ub in PD pathology remain important questions to be addressed.

A large study of PD patient fibroblasts identified distinct subsets of SPD patients with mitochondrial or lysosomal dysfunction [72]. Specifically, a subset of patient fibroblasts demonstrated altered complex I and IV protein levels and these deficits were enhanced after direct differentiation of dopaminergic neurons [72]. In addition, we have demonstrated that bioenergetics are altered in peripheral blood mononuclear cells from sPD patients [73]. These studies further suggest that systemic mitochondrial dysfunction, combined with the high metabolic demand of dopaminergic neurons, leads to the preferential vulnerability of dopaminergic neurons in PD (Box 3).

Common mechanisms between monogenic and sporadic disease have been noted, such as impaired degradation of MIRO at the mitochondrial surface, which is also observed in LRRK2 models $[51,74]$, and these overlaps are likely key to understanding convergent pathways in PD subgroups. Highlighting this, SNPs in a number of mitochondrial genes have been associated with the age of onset of PD [75], further suggesting a substantial role for mitochondria in SPD aetiology and the need for multiple pathways to converge to initiate disease pathology.

\section{Therapeutics targeting mitochondrial dysfunction}

Loss of mitochondrial quality control could substantially contribute to the high susceptibility of SNpc neurons to neurodegeneration in PD (Box 3). Recent advances in our understanding of mitochondrial homeostasis and damage in PD has led to the identification of a number of potential therapeutic avenues to promote the clearance of old or damaged mitochondria in both PD and other diseases involving mitochondrial dysfunction.

Small molecule activators of PINK1 and parkin, or inhibitors of USP30 and pSer65Ub phosphatases, are promising therapeutic targets for enhancing mitophagy in PD. Similarly, although less defined, USP8, USP14, and USP15 inhibitors represent promising routes to upregulating mitophagy $[20,76,77]$.

Direct activation of PINK1 has been demonstrated by kinetin triphosphate KTP [78] and further development of bioavailable KTP precursors is underway [79]. In addition, two smallmolecule activators of PINK1 which are structurally distinct from KTP have been identified by high-throughput screening and have been validated in both dopaminergic neurons and PINK1 knockdown Drosophila [80]. An orthogonal approach of directly expressing recombinant 
parkin engineered for increased solubility and cell permeability has shown promise in protecting cells from both toxin and $\alpha$-synuclein challenge [81].

Direct targeting of proteins impacted by autosomal dominant PD mutations offers a strategy to expand therapy beyond PINK1/PRKN patients. Encouragingly, two independent studies have demonstrated that LRRK2 kinase inhibitors are able to correct impaired RAB10optineurin and parkin-Drp1 interactions in LRRK2 mutant cells $[53,54]$. The increased LRRK2 kinase activity in idiopathic PD has also been shown in animal models in which LRRK2 inhibition corrected mitochondrial and lysosomal dysfunction [82, 83]. LRRK2 inhibitors currently in clinical trials could therefore hold great promise for correcting mitochondrial dysfunction and mitophagy defects beyond patients with LRRK2 mutations. Similarly, the effects of augmentation of GCase activity on peripheral mitochondrial dysfunction using small molecule chaperones such as ambroxol, TFEB activators or recombinant GCase-like enzyme such as Cerezyme, will be of great interest. Moreover, nicotinamide riboside has been demonstrated to correct mitochondrial phenotype in iPSC-derived neurons carrying mutations in GBA, and in Drosophila models [57]. In addition, several small molecules, such as the repositionable drug ursodeoxycholic acid, have been demonstrated to augment mitochondrial complex activity and ATP production and to be beneficial in PD models including, importantly, in SPD fibroblasts [72].

\section{Concluding remarks}

Overall, recent progress has advanced our knowledge of the mechanisms regulating PINK1/parkin-mediated mitophagy. Further understanding the relative contributions of this pathway to basal and stress-induced mitophagy in dopaminergic neurons, when compared to non-PINK1/parkin-dependent mechanisms, will aid in the development of potential therapeutic activators of this pathway. Similarly, it is imperative to clarify the role of PINK1/parkin-dependent mitophagy in non-neuronal cells, such as astrocytes and microglia in which this pathway appears to be more active, in order to determine how deficits in mitochondrial quality control in glia contribute to the pathology of PD (see Outstanding Questions).

Given the multiple cellular mechanisms that are dysfunctional in LRRK2, SNCA, and GBArelated $P D$, enhancing mitophagy and improving mitochondrial health may only partially rescue dopaminergic cell loss in non-PINK1/PRKN-related diseases. Furthermore, the expression of PD risk genes, particularly $L R R K 2$, is not homogenous across brain regions or cell types, raising the possibility of an important role for non-neuronal cells in suppressing mitochondrial dysfunction through mechanisms, such as trans-mitochondrial degradation (transmitophagy) or by the propagation of mitochondrial damage in either a cell-autonomous manner or through inflammatory processes such as MITAP or STING-activation. Similarly, the roles of genes such as CHCHD2 and VPS35 in mitochondrial dysfunction in dopaminergic 
431 neurons and their contribution (if any) to disease in the wider PD population remains to be

432 elucidated. Regardless of the genetic background of patients, the role(s) that increased LRRK2

433 kinase activity and $\alpha$-synuclein aggregation play in damaging mitochondria and its impact of

434 mitophagy is likely key in understanding convergent mechanisms in PD.

435

436 Investigation of novel PD-causing genes with mitochondrial roles, combined with the

437 investigation of mitochondrial deficits in sporadic PD, will identify novel and overlapping

438 mechanisms of dysfunction to enhance our understanding of the role of mitochondrial

439 dysfunction in the disease process. Given the widespread prevalence of mitochondrial

440 damage in a range of diseases, these mechanistic insights and the resulting therapeutic

441 opportunities may also have utility beyond PD.

442

443

444

445

446

\section{Acknowledgements}

448 ABM, MW, BJR and RW-M are supported by the BMS/Celgene Oxford DUB Alliance. This work 449 was also supported by the Monument Trust Discovery Award from Parkinson's UK (J-1403)

450 and by the Intramural Research Program of the NIH, NINDS. Figure 2 was created using 451 Biorender. 
1. Kitada, T. et al. (1998) Mutations in the parkin gene cause autosomal recessive juvenile parkinsonism. Nature 392 (6676), 605-8.

2. Valente, E.M. et al. (2001) Localization of a novel locus for autosomal recessive early-onset parkinsonism, PARK6, on human chromosome 1p35-p36. Am J Hum Genet 68 (4), 895-900.

3. Narendra, D.P. et al. (2010) PINK1 is selectively stabilized on impaired mitochondria to activate Parkin. PLoS Biol 8, e1000298.

4. Pickles, S. et al. (2018) Mitophagy and Quality Control Mechanisms in Mitochondrial Maintenance. Curr Biol 28 (4), R170-r185.

5. Swatek, K.N. et al. (2019) Insights into ubiquitin chain architecture using Ub-clipping. Nature 572 (7770), 533537.

6. Wauer, T. et al. (2015) Mechanism of phospho-ubiquitin-induced PARKIN activation. Nature 524 (7565), 3704.

7. Gladkova, C. et al. (2018) Mechanism of parkin activation by PINK1. Nature 559 (7714), 410-414.

8. Harper, J.W. et al. (2018) Building and decoding ubiquitin chains for mitophagy. Nature Reviews Molecular Cell Biology 19 (2), 93-108.

9. Hou, X. et al. (2018) Age- and disease-dependent increase of the mitophagy marker phospho-ubiquitin in normal aging and Lewy body disease. Autophagy 14 (8), 1404-1418.

10. Fiesel, F.C. et al. (2015) (Patho-)physiological relevance of PINK1-dependent ubiquitin phosphorylation. EMBO reports 16 (9), 1114-1130.

11. Schubert, A.F. et al. (2017) Structure of PINK1 in complex with its substrate ubiquitin. Nature 552 (7683), 51-56.

12. Potting, C. et al. (2018) Genome-wide CRISPR screen for PARKIN regulators reveals transcriptional repression as a determinant of mitophagy. Proceedings of the National Academy of Sciences 115 (2), E180-E189. 13. Hoshino, A. et al. (2019) The ADP/ATP translocase drives mitophagy independent of nucleotide exchange. Nature 575 (7782), 375-379.

14. Bhupana, J.N. et al. (2020) Gas7 knockout affects PINK1 expression and mitochondrial dynamics in mouse cortical neurons. FASEB BioAdvances 2 (3), 166-181.

15. Soutar, M.P.M. et al. (2018) AKT signalling selectively regulates PINK1 mitophagy in SHSY5Y cells and human iPSC-derived neurons. Scientific Reports 8 (1), 8855.

16. Rizza, S. et al. (2018) S-nitrosylation drives cell senescence and aging in mammals by controlling mitochondrial dynamics and mitophagy. Proceedings of the National Academy of Sciences 115 (15), E3388E3397.

17. Bendall, J.K. et al. (2005) Stoichiometric Relationships Between Endothelial Tetrahydrobiopterin, Endothelial NO Synthase (eNOS) Activity, and eNOS Coupling in Vivo: Insights From Transgenic Mice With EndothelialTargeted GTP Cyclohydrolase 1 and eNOS Overexpression. Circ Res 97 (9), 864-871.

18. Chung, K.K. et al. (2004) S-nitrosylation of parkin regulates ubiquitination and compromises parkin's protective function. Science 304 (5675), 1328-31.

19. Oh, C.-K. et al. (2017) S-Nitrosylation of PINK1 Attenuates PINK1/Parkin-Dependent Mitophagy in hiPSCBased Parkinson's Disease Models. Cell Reports 21 (8), 2171-2182.

20. Chakraborty, J. et al. (2018) USP14 inhibition corrects an in vivo model of impaired mitophagy. EMBO Mol Med 10 (11).

21. Cunningham, C.N. et al. (2015) USP30 and parkin homeostatically regulate atypical ubiquitin chains on mitochondria. Nat Cell Biol 17 (2), 160-9.

22. Rusilowicz-Jones, E.V. et al. (2020) USP30 sets a trigger threshold for PINK1-PARKIN amplification of mitochondrial ubiquitylation. Life Sci Alliance 3 (8), e202000768.

23. Sato, Y. et al. (2017) Structural basis for specific cleavage of Lys6-linked polyubiquitin chains by USP30. Nat Struct Mol Biol 24 (11), 911-919.

24. Gersch, M. et al. (2017) Mechanism and regulation of the Lys6-selective deubiquitinase USP30. Nat Struct Mol Biol 24 (11), 920-930.

25. Marcassa, E. et al. (2018) Dual role of USP30 in controlling basal pexophagy and mitophagy. EMBO reports 19, e45595.

26. Bingol, B. et al. (2014) The mitochondrial deubiquitinase USP30 opposes parkin-mediated mitophagy. Nature 510 (7505), 370-5.

27. Ordureau, A. et al. (2020) Global Landscape and Dynamics of Parkin and USP30-Dependent Ubiquitylomes in iNeurons during Mitophagic Signaling. Mol Cell 77 (5), 1124-1142 e10.

28. Phu, L. et al. (2020) Dynamic Regulation of Mitochondrial Import by the Ubiquitin System. Mol Cell 77 (5), $1107-1123$ e10.

511 29. Wauer, T. et al. (2015) Ubiquitin Ser65 phosphorylation affects ubiquitin structure, chain assembly and 
hydrolysis. EMBO J 34 (3), 307-25.

513 30. Wang, L. et al. (2018) PTEN-L is a novel protein phosphatase for ubiquitin dephosphorylation to inhibit PINK1-Parkin-mediated mitophagy. Cell Research 28 (8), 787-802.

515 31. Wall, C.E. et al. (2019) PPEF2 Opposes PINK1-Mediated Mitochondrial Quality Control by

516 Dephosphorylating Ubiquitin. Cell Rep 29 (10), 3280-3292 e7.

517 32. Li, G. et al. (2018) PTEN $\alpha$ regulates mitophagy and maintains mitochondrial quality control. Autophagy 14 518 (10), 1742-1760.

519 33. Wall, C.E. et al. (2019) PPEF2 Opposes PINK1-Mediated Mitochondrial Quality Control by Dephosphorylating Ubiquitin. Cell Reports 29 (10), 3280-3292.e7.

34. Barodia, S.K. et al. (2019) PINK1 phosphorylates ubiquitin predominantly in astrocytes. NPJ Parkinsons Dis $5,29$.

35. Lee, Y. et al. (2017) PINK1 Primes Parkin-Mediated Ubiquitination of PARIS in Dopaminergic Neuronal Survival. Cell Rep 18 (4), 918-932.

36. Noda, S. et al. (2020) Loss of Parkin contributes to mitochondrial turnover and dopaminergic neuronal loss in aged mice. Neurobiol Dis 136, 104717.

37. Bogetofte, H. et al. (2019) Perturbations in RhoA signalling cause altered migration and impaired neuritogenesis in human iPSC-derived neural cells with PARK2 mutation. Neurobiol Dis 132, 104581.

38. Martinez, A. et al. (2017) Quantitative proteomic analysis of Parkin substrates in Drosophila neurons. Molecular Neurodegeneration 12 (1), 29.

39. Okarmus, J. et al. (2020) Lysosomal perturbations in human dopaminergic neurons derived from induced pluripotent stem cells with PARK2 mutation. Sci Rep 10 (1), 10278.

40. McLelland, G.L. et al. (2018) Mfn2 ubiquitination by PINK1/parkin gates the p97-dependent release of ER from mitochondria to drive mitophagy. Elife 7, e32866.

41. Creed, R.B. et al. (2019) Basal and Evoked Neurotransmitter Levels in Parkin, DJ-1, PINK1 and LRRK2 Knockout Rat Striatum. Neuroscience 409, 169-179.

42. Sliter, D.A. et al. (2018) Parkin and PINK1 mitigate STING-induced inflammation. Nature 561 (7722), 258262.

43. Matheoud, D. et al. (2019) Intestinal infection triggers Parkinson's disease-like symptoms in Pink1(-/-) mice. Nature 571 (7766), 565-569.

44. Di Maio, R. et al. (2016) $\alpha$-Synuclein binds to TOM20 and inhibits mitochondrial protein import in Parkinson's disease. Science Translational Medicine 8 (342), 342ra78-342ra78.

45. Zambon, F. et al. (2019) Cellular alpha-synuclein pathology is associated with bioenergetic dysfunction in Parkinson's iPSC-derived dopamine neurons. Hum Mol Genet 28, 2001-2013.

46. Jacobs, D. et al. (2019) Probing Membrane Association of $\alpha$-Synuclein Domains with VDAC Nanopore Reveals Unexpected Binding Pattern. Scientific Reports 9 (1), 4580.

47. Ludtmann, M.H.R. et al. (2018) $\alpha$-synuclein oligomers interact with ATP synthase and open the permeability transition pore in Parkinson's disease. Nature Communications 9 (1), 2293.

48. Wang, X. et al. (2019) Pathogenic alpha-synuclein aggregates preferentially bind to mitochondria and affect cellular respiration. Acta Neuropathologica Communications 7 (1), 41.

49. Mahul-Mellier, A.L. et al. (2020) The process of Lewy body formation, rather than simply alpha-synuclein fibrillization, is one of the major drivers of neurodegeneration. Proc Natl Acad Sci U S A 117 (9), 4971-4982.

50. Shahmoradian, S.H. et al. (2019) Lewy pathology in Parkinson's disease consists of crowded organelles and lipid membranes. Nature Neuroscience 22 (7), 1099-1109.

51. Hsieh, C.-H. et al. (2016) Functional Impairment in Miro Degradation and Mitophagy Is a Shared Feature in Familial and Sporadic Parkinson's Disease. Cell Stem Cell 19, 709.

52. Godena, V.K. et al. (2014) Increasing microtubule acetylation rescues axonal transport and locomotor deficits caused by LRRK2 Roc-COR domain mutations. Nat Commun 5, 5245.

53. Bonello, F. et al. (2019) LRRK2 impairs PINK1/Parkin-dependent mitophagy via its kinase activity: pathologic insights into Parkinson's disease. Hum Mol Genet 28 (10), 1645-1660.

54. Wauters, F. et al. (2019) LRRK2 mutations impair depolarization-induced mitophagy through inhibition of mitochondrial accumulation of RAB10. Autophagy, 1-20.

55. West, A.B. et al. (2014) Differential LRRK2 expression in the cortex, striatum, and substantia nigra in transgenic and nontransgenic rodents. J Comp Neurol 522 (11), 2465-80.

56. $\mathrm{Li}$, H. et al. (2019) Mitochondrial dysfunction and mitophagy defect triggered by heterozygous GBA mutations. Autophagy 15 (1), 113-130.

57. Schondorf, D.C. et al. (2018) The NAD+ Precursor Nicotinamide Riboside Rescues Mitochondrial Defects and Neuronal Loss in iPSC and Fly Models of Parkinson's Disease. Cell Rep 23 (10), 2976-2988.

58. Funayama, M. et al. (2015) CHCHD2 mutations in autosomal dominant late-onset Parkinson's disease: a genome-wide linkage and sequencing study. The Lancet Neurology 14 (3), 274-282.

571 59. Liu, Y.T. et al. (2020) Loss of CHCHD2 and CHCHD10 activates OMA1 peptidase to disrupt mitochondrial 
cristae phenocopying patient mutations. Hum Mol Genet 29, 1547-1567.

60. Huang, X. et al. (2019) CHCHD2 accumulates in distressed mitochondria and facilitates oligomerization of CHCHD10. Hum Mol Genet 28 (2), 349.

61. Cornelissen, T. et al. (2020) CHCHD2 harboring the Parkinson's disease-linked T61I mutation precipitates inside mitochondria and induces precipitation of wild-type CHCHD2. Hum Mol Genet 29, 1096-1106.

62. Meng, H. et al. (2017) Loss of Parkinson's disease-associated protein CHCHD2 affects mitochondrial crista structure and destabilizes cytochrome c. Nat Commun 8, 15500.

63. Ikeda, A. et al. (2019) Mutations in CHCHD2 cause alpha-synuclein aggregation. Hum Mol Genet 28 (23), 3895-3911.

64. Mencacci, N.E. et al. (2014) Parkinson's disease in GTP cyclohydrolase 1 mutation carriers. Brain 137, 248092.

65. Ryan, B.J. et al. (2014) alpha-Synuclein and mitochondrial bioenergetics regulate tetrahydrobiopterin levels in a human dopaminergic model of Parkinson disease. Free Radic Biol Med 67, 58-68.

66. Bailey, J. et al. (2017) A novel role for endothelial tetrahydrobiopterin in mitochondrial redox balance. Free Radical Biology and Medicine 104, 214-225.

67. Wang, W. et al. (2016) Parkinson's disease-associated mutant VPS35 causes mitochondrial dysfunction by recycling DLP1 complexes. Nature medicine 22 (1), 54-63.

68. Braschi, E. et al. (2010) Vps35 mediates vesicle transport between the mitochondria and peroxisomes. Curr Biol 20 (14), 1310-5.

69. Jimenez-Orgaz, A. et al. (2018) Control of RAB7 activity and localization through the retromer-TBC1D5 complex enables RAB7-dependent mitophagy. The EMBO Journal 37 (2), 235-254.

70. Inoshita, T. et al. (2017) Vps35 in cooperation with LRRK2 regulates synaptic vesicle endocytosis through the endosomal pathway in Drosophila. Human Molecular Genetics 26 (15), 2933-2948.

71. Shiba-Fukushima, K. et al. (2017) Evidence that phosphorylated ubiquitin signaling is involved in the etiology of Parkinson's disease. Human Molecular Genetics 26 (16), 3172-3185.

72. Carling, P.J. et al. (2020) Deep phenotyping of peripheral tissue facilitates mechanistic disease stratification in sporadic Parkinson's disease. Progress in Neurobiology 187, 101772.

73. Smith, A.M. et al. (2018) Mitochondrial dysfunction and increased glycolysis in prodromal and early Parkinson's blood cells. Mov Disord 33 (10), 1580-1590.

74. Shaltouki, A. et al. (2018) Alpha-synuclein delays mitophagy and targeting Miro rescues neuron loss in Parkinson's models. Acta Neuropathologica 136, 607-620.

75. Billingsley, K.J. et al. (2019) Mitochondria function associated genes contribute to Parkinson's Disease risk and later age at onset. npj Parkinson's Disease 5 (1), 8.

76. Durcan, T.M. et al. (2014) USP8 regulates mitophagy by removing K6-linked ubiquitin conjugates from parkin. The EMBO journal 33 (21), 2473-2491.

77. Teyra, J. et al. (2019) Structural and Functional Characterization of Ubiquitin Variant Inhibitors of USP15. Structure 27 (4), 590-605.e5.

78. Hertz, N.T. et al. (2013) A neo-substrate that amplifies catalytic activity of parkinson's-disease-related kinase PINK1. Cell 154 (4), 737-47.

612 Mutated in Parkinson's Disease. Chembiochem 19 (23), 2433-2437.

613 80. Shiba-Fukushima, K. et al. (2020) A cell-based high-throughput screening identified two compounds that

614 enhance PINK1-Parkin signaling. iScience, 101048.

615 81. Chung, E. et al. (2020) Intracellular delivery of Parkin rescues neurons from accumulation of damaged mitochondria and pathological $\alpha$-synuclein. Science Advances 6 (18), eaba1193.

82. Sanyal, A. et al. (2020) Lysosome and Inflammatory Defects in GBA1-Mutant Astrocytes Are Normalized by LRRK2 Inhibition. Movement Disorders 35 (5), 760-773.

619 83. Rocha, E.M. et al. (2020) LRRK2 inhibition prevents endolysosomal deficits seen in human Parkinson's disease. Neurobiol Dis 134, 104626.

84. McWilliams, T.G. et al. (2016) mito-QC illuminates mitophagy and mitochondrial architecture in vivo. Journal of Cell Biology 214 (3), 333-345.

85. Katayama, H. et al. (2011) A sensitive and quantitative technique for detecting autophagic events based on lysosomal delivery. Chem Biol 18 (8), 1042-52.

86. Katayama, H. et al. (2020) Visualizing and Modulating Mitophagy for Therapeutic Studies of Neurodegeneration. Cell 181 (5), 1176-1187 e16.

87. McWilliams, T.G. et al. (2018) Basal Mitophagy Occurs Independently of PINK1 in Mouse Tissues of High Metabolic Demand. Cell Metab 27 (2), 439-449 e5.

88. Lee, J.J. et al. (2018) Basal mitophagy is widespread in Drosophila but minimally affected by loss of Pink1 or parkin. J Cell Biol 217 (5), 1613-1622.

631 89. Cornelissen, T. et al. (2018) Deficiency of parkin and PINK1 impairs age-dependent mitophagy in Drosophila. 
eLife 7, e35878.

633 90. Vincow, E.S. et al. (2013) The PINK1-Parkin pathway promotes both mitophagy and selective respiratory chain turnover in vivo. Proceedings of the National Academy of Sciences 110 (16), 6400-6405.

91. Diot, A. et al. (2015) A novel quantitative assay of mitophagy: Combining high content fluorescence microscopy and mitochondrial DNA load to quantify mitophagy and identify novel pharmacological tools against pathogenic heteroplasmic mtDNA. Pharmacological Research 100, 24-35.

92. Li, J. et al. (2015) Mitochondrial outer-membrane E3 ligase MUL1 ubiquitinates ULK1 and regulates seleniteinduced mitophagy. Autophagy 11 (8), 1216-1229.

93. Szargel, R. et al. (2016) The PINK1, synphilin-1 and SIAH-1 complex constitutes a novel mitophagy pathway. Human Molecular Genetics 25 (16), 3476-3490.

94. Burchell, V.S. et al. (2013) The Parkinson's disease-linked proteins Fbxo7 and Parkin interact to mediate mitophagy. Nat Neurosci 16 (9), 1257-65.

95. Zhou, Z.D. et al. (2015) F-box protein 7 mutations promote protein aggregation in mitochondria and inhibit mitophagy. Human Molecular Genetics 24 (22), 6314-6330.

96. Villa, E. et al. (2018) No Parkin Zone: Mitophagy without Parkin. Trends in Cell Biology.

97. Chen, G. et al. (2014) A Regulatory Signaling Loop Comprising the PGAM5 Phosphatase and CK2 Controls Receptor-Mediated Mitophagy. Molecular Cell 54 (3), 362-377.

98. Chen, Z. et al. (2017) Mitochondrial E3 ligase MARCH5 regulates FUNDC1 to fine-tune hypoxic mitophagy. EMBO reports 18 (3), 495-509.

99. McLelland, G.L. et al. (2014) Parkin and PINK1 function in a vesicular trafficking pathway regulating mitochondrial quality control. EMBO J 33 (4), 282-295.

100. Todkar, K. et al. (2020) Mitochondria-Derived Vesicles prevent inflammation by controlling the inclusion of mitochondrial proteins into extracellular vesicles. bioRxiv, 2020.03.13.989814.

101. Ryan, T.A. et al. (2020) Tollip coordinates Parkin-dependent trafficking of mitochondrial-derived vesicles. Embo j 39 (11), e102539.

102. Morales, I. et al. (2020) Neuroglial transmitophagy and Parkinson's disease. Glia 68, 2277-2299.

103. Bolam, J.P. and Pissadaki, E.K. (2012) Living on the edge with too many mouths to feed: why dopamine neurons die. Mov Disord 27 (12), 1478-83.

104. Caudle, W.M. et al. (2007) Reduced vesicular storage of dopamine causes progressive nigrostriatal neurodegeneration. J Neurosci 27 (30), 8138-48.

105. Graves, S.M. et al. (2020) Dopamine metabolism by a monoamine oxidase mitochondrial shuttle activates the electron transport chain. Nature Neuroscience 23 (1), 15-20.

106. Guzman, J.N. et al. (2018) Systemic isradipine treatment diminishes calcium-dependent mitochondrial oxidant stress. The Journal of clinical investigation 128 (6), 2266-2280.

107. Ludtmann, M.H.R. and Abramov, A.Y. (2018) Mitochondrial calcium imbalance in Parkinson's disease. Neuroscience Letters 663, 86-90.

108. Dölle, C. et al. (2016) Defective mitochondrial DNA homeostasis in the substantia nigra in Parkinson disease. Nature Communications 7 (1), 13548.

109. Mokretar, K. et al. (2018) Somatic copy number gains of $\alpha$-synuclein (SNCA) in Parkinson's disease and multiple system atrophy brains. Brain 141 (8), 2419-2431.

110. McFarland, M.A. et al. (2008) Proteomics analysis identifies phosphorylation-dependent alpha-synuclein protein interactions. Mol Cell Proteomics 7 (11), 2123-37. 
677

678

679

680

681

682

683

684

685

686

687

688

689

690

691

692

693

694

695

696

697

698

699

700

701

702

703

704

705

706

707

708

709

710

711

712

713

714

715

716

717

718

719

720

721

722

$\boldsymbol{\alpha}$-synuclein: the major component of Lewy bodies and a protein with diverse roles in cellular biology; mutations in $\alpha$-synuclein increase the propensity for the protein to aggregate and cause autosomal dominant familial Parkinson's disease (PD)

Cyanide m-chlorophenyl hydrazine (CCCP): is a protonophore and a potent mitochondrial uncoupler that depolarizes the mitochondrial membrane and induces mitophagy; CCCP is extensively used in the study of PINK1/Parkin-dependent mitophagy

CHCHD2: a mitochondrial protein and familial PD risk gene which regulates mitochondrial function

Dopaminergic neurons: neurons which release the neurotransmitter dopamine; the midbrain dopaminergic neurons in the substantia nigra pars compacta (SNpc) are preferentially vulnerable in PD, whereas the neighbouring dopaminergic neurons of the ventral tegmental area (VTA) are relatively spared.

Deubiquitylases (DUBs): a large group of proteins that remove ubiquitin (Ub) chains from proteins; DUBs such as USP8, USP14, USP15, USP35, and most prominently USP30 have been found to regulate mitophagy by antagonising parkin activity

Lewy bodies: intracellular protein aggregates comprised of misfolded proteins of which $\alpha$-synuclein is a prominent component; Lewy bodies are the defining pathological feature of post-mortem PD brain

LRRK2: leucine-rich repeat kinase 2 (LRRK2) is a widely expressed multidomain kinase; mutations in LRRK2 are the most common form of autosomal dominant PD

Mitophagy: a form of selective autophagy targeting damaged mitochondria making use of two major degradation systems: autophagy and the ubiquitin-proteasome system; mitophagy acts as a mitochondrial quality-control mechanism

Parkin: an E3 Ub ligase catalysing the attachment of Ub chains to substrate proteins; after activation by PINK1, parkin ubiquitinates outer mitochondrial membrane proteins and mediates the clearance of damaged mitochondria, and mutations in PRKN cause autosomal recessive PD

Parkinson's disease (PD): a progressive, neurodegenerative disease characterized by both motor and non-motor symptoms; pathologically, the disease is characterized by the aggregation of $\alpha$-synuclein into Lewy bodies and the preferential degeneration of dopaminergic neurons of the SNpc

PINK1: PTEN-induced putative kinase 1 (PINK1) is a serine/threonine-protein kinase playing a central role in mitophagy following mitochondrial membrane depolarization phosphorylating both parkin and Ub; mutations in PINK1 cause autosomal recessive PD pSer65Ub: PINK1 phosphorylates the serine 65 residue on Ub chains on the outer mitochondrial membrane forming phospho-serine65 Ub (pSer65Ub); pSer65Ub plays a crucial role in the amplification of the PINK1/parkin pathway

Substantia nigra pars compacta (SNpc): the dopaminergic nigrostriatal neurons arising from the SNpc release dopamine in the striatum modulating motor activity; the SNpc A9 region is the major site of dopaminergic neuron loss in PD

TOM complex: the translocase of the outer mitochondrial membrane (TOM) is a complex of proteins (e.g. TOM 20 and TOM40) that regulate protein transport across the outer mitochondrial membrane

Trans-mitophagy: degradation of mitochondria in a non-cell-autonomous manner, e.g. by glial cells 
Ubiquitin (Ub): a small protein that can post-translationally modify proteins, influencing their function and targeting them for degradation Ventral tegmental area (VTA): a dopaminergic region of the midbrain neighbouring the SNpc. The VTA A10 dopaminergic neurons are relatively spared in PD 

relevance to PD

731 The majority of studies of PINK1/parkin-dependent mitophagy have relied on chemical agents

732 to inhibit mitochondrial function. Use of the ionophore cyanide $\mathbf{m}$-chlorophenyl hydrazine 733 (CCCP), or a combination of the ATP synthase inhibitor oligomycin and the complex III 734 inhibitor antimycin A, are the most common agents used to activate PINK1/parkin-dependent mitophagy. Whilst informative, the large-scale loss of mitochondrial function in the cell after such chemical inhibition represents supra-pathophysiological conditions. In addition, much research has relied on expression/overexpression of parkin in order to observe mitophagyrelated phenomena such as parkin translocation, TOM20 loss, and lysosomal cargo delivery. The identification of the pathophysiologically-relevant activators of PINK1/parkin mitophagy remains to be elucidated.

The field has been advanced by tools to measure the delivery of $\mathrm{pH}$-sensitive proteins fused to mitochondrial cargo destined for lysosomes such as mitoQC [84], mtKeima [85], and recently, mito-SRAI [86]. Data from Drosophila expressing mitoQC or mtKeima and from mouse dopaminergic neurons expressing mitoQC have demonstrated basal mitophagy is not affected by the loss of PINK1 or parkin $[87,88]$. These data seemingly contradict experiments using mtKeima demonstrating a role for PINK1 in basal mitophagy [89], leaving the relative contribution of the PINK1/parkin pathway to basal mitophagy unclear. However, given the localization of mitoQC to the outer mitochondrial membrane (OMM) and mtKeima or mitoSRAI to the mitochondrial matrix, the relative contributions of OMM protein degradation by proteasome (or MDVs) in different organisms and cell types, may be responsible for the discrepancy [86], suggesting multiple tools and approaches may be required to gain consensus.

Recently, modified versions of mitoQC, targeted to the matrix with a Flag-V5 processing sequence ( $m t x-X L)$, and mtKeima, with an additional Flag-V5 epitope ( $m t x-K e i m a{ }^{X L}$ ), have been generated [27], potentially closing the subtle methodological gaps between these two tools. Independent methods of assessing mitophagy, such as mitochondrial protein turnover using mass spectrometry, which identified both Atg7-dependent and independent roles of PINK1-parkin in Drosophila and a reduction of mitochondrial heteroplasmy with PINK1/parkin overexpression [90], suggest PINK1/parkin can play a role. In addition, assays such as TOM20/LC3 co-localisation provide alternative methods to assess mitochondrial turnover [91]. 

conclusions in cell models and in vivo. 
While the canonical PINK1/parkin pathway is linked to Parkinson's disease (PD) through familial genetics, additional PINK1/parkin-independent mechanisms of degradation of damaged mitochondria in mammalian cells are also important in the disease.

A number of PINK1-independent pathways mediated by outer mitochondrial membrane (OMM) proteins that can interact with Atg8 homologs at the autophagosome through an LIRinteracting domain (LC3-interacting domain) have been identified, including BNIP3 (BCl2/adenovirus E1B 19-kDa interacting protein 3), NIX (BNIP3-like; BNIP3L), FUNDC1 (FUN14 Domain Containing 1), and AMBRA1. Additionally, a number of E3 Ub ligases other than parkin have been demonstrated to ubiquitinate OMM proteins including MUL1 [92] and SIAH1 [93], resulting in receptor-mediated mitophagy. The PD-associated gene FBXO7 (PARK15) encodes an adaptor in the FBXO7-SCF E3-ligase complex and facilitates parkin translocation to mitochondria and mitophagy [94]. Mutations in FBXO7 impair FBXO7-parkin interactions resulting in mitochondrial protein misfolding and impaired mitophagy [94, 95]

Post-translational regulation of OMM-LIR domain proteins occurs in response to a diverse range of stimuli beyond the loss of membrane potential $\left(\psi_{\mathrm{m}}\right)$ (as reviewed in [96]). For example, FUNDC1 phosphorylation status at the $\mathrm{N}$-terminal LIR domain regulates its interaction with LC3 in response to the loss of $\psi_{\mathrm{m}}$ or hypoxia with the phosphoglycerate mutase family member 5 (PGAM5) acting as a phosphatase allowing the interaction of FUNDC1 with LC3 [97]. Interestingly, MARCH-5 ubiquitinates FUNDC1 targeting it for degradation [98], suggesting a coordinated interplay between the USP30-PINK1-parkin axis and (PINK1/parkin-independent) FUNDC1.

Mitochondrial derived vesicles (MDVs) are formed in response to mitochondrial damage, specifically sequestering damaged mitochondrial cargo into vesicles which are delivered to the lysosome [99]. This process is dependent on PINK1 and parkin but it is independent of canonical macroautophagy machinery [99]. Parkin, via Tollip, has recently been hypothesised to act as a switch controlling the trafficking of MDVs towards the lysosome or towards extracellular vesicles facilitated by sorting nexin 9, OPA-1 and VPS35 [68, 100, 101]. The role of MDV formation in mitochondrial homeostasis is under investigation, with the incorporation of immunogenic mitochondrial components such as mitochondrial DNA (mtDNA) into MDVs demonstrated to be less immunogenic than their release which activates the inflammasome/NFKB pathway [100]. Furthermore, there may be a non-cell-autonomous component of neuronal mitophagy, particularly in distal axons, mediated by MDVs or spheroid-like structures incorporating damaged mitochondria [102].

Understanding mitophagy pathways outside of the canonical PINK1-parkin pathway will allow greater insight into the compensation that occurs in PD patients with PINK1/parkin mutations 
809 and the specific stimuli which trigger the PINK1-parkin independent pathway in dopaminergic 810 neurons. 
813 The physiology, biochemistry, and anatomy of the A9 dopaminergic neurons of the substantia

814 nigra pars compacta ( $\mathrm{SNpc}$ ) may underpin the preferential vulnerability of these neurons in

815 Parkinson's disease (PD) compared to other neuronal types, such as neighbouring A10

816 dopaminergic neurons of the Ventral tegmental area (VTA). Susceptibility factors of SNpc

817 neurons include their extensive axonal arbour and consequent high bioenergetic demand

818 [103], the presence of dopamine, persistent $\mathrm{Ca}^{2+}$ flux driven by pacemaking activity, and the

819 post-mitotic nature of neurons which render them susceptible to the accumulation of somatic

820 mutations.

821

822 Dopamine can undergo auto-oxidation resulting in the generation of hydrogen peroxide, 823 which can cause oxidative stress through the Fenton reaction. Therefore, any impairment of 824 dopamine sequestration into vesicles results in increased dopamine toxicity [104]. It has 825 recently been observed that dopamine can serve as a source of electrons in dopaminergic 826 neurons feeding into complex IV, driving mitochondrial membrane potential and ATP 827 production during bursts of dopaminergic neuron activity in a feed-forward mechanism [105]. 828 Whilst this observation provides an attractive hypothesis for increased bioenergetic demand 829 during synaptic release/recycling, this mechanism may also contribute to ROS generation and 830 mitochondrial damage through dopamine auto-oxidation or electron leak. Indeed, evidence 831 suggests that dopaminergic SNpc neurons undergo an increased rate of mitophagy and 832 mitochondrial turnover relative to dopaminergic neurons in the VTA [106].

833

834 High fluxes of cytosolic $\mathrm{Ca}^{2+}$ through L-type calcium channels during neuronal activity are a 835 feature of dopaminergic neurons of the SNpc in addition to efflux via the $\mathrm{Na}^{+} / \mathrm{Ca}^{2+} / \mathrm{Li}^{+}$ 836 exchanger NCLX (as reviewed in [107]). Mitochondria and mitochondrial-ER contact sites play 837 a key role in buffering cytosolic $\mathrm{Ca}^{2+}$ fluxes with the CaV1 channel inhibitor isradipine having 838 been shown to decrease mitochondrial oxidative stress in nigral dopaminergic neurons 839 without altering pacemaking activity [106].

840

841 Accumulation of somatic mutations and genetic mosaicism in the midbrain of PD patients may 842 result from the damage of mitochondrial and nuclear DNA by ROS. It has been shown that 843 mtDNA copy number is increased during healthy ageing of the SNpc whereas nigral neurons 844 in PD patients have a depletion of mtDNA copy number in the absence of increased rates of 845 mtDNA damage, suggesting mtDNA homeostasis is impaired in PD [108]. In addition, PD 846 patients may have an increased SNCA copy number in the nucleus [109]. Somatic loss of 847 function mutations or increased CNVs in genes such as SNCA may provide an alternative route 848 to mitochondrial toxicity or protein aggregation. 
850 Together, these data suggest mechanisms by which the unique neuronal physiology of SNpc 851 dopaminergic neurons play a key role in propagating and being affected by mitochondrial 852 dysfunction in PD. 


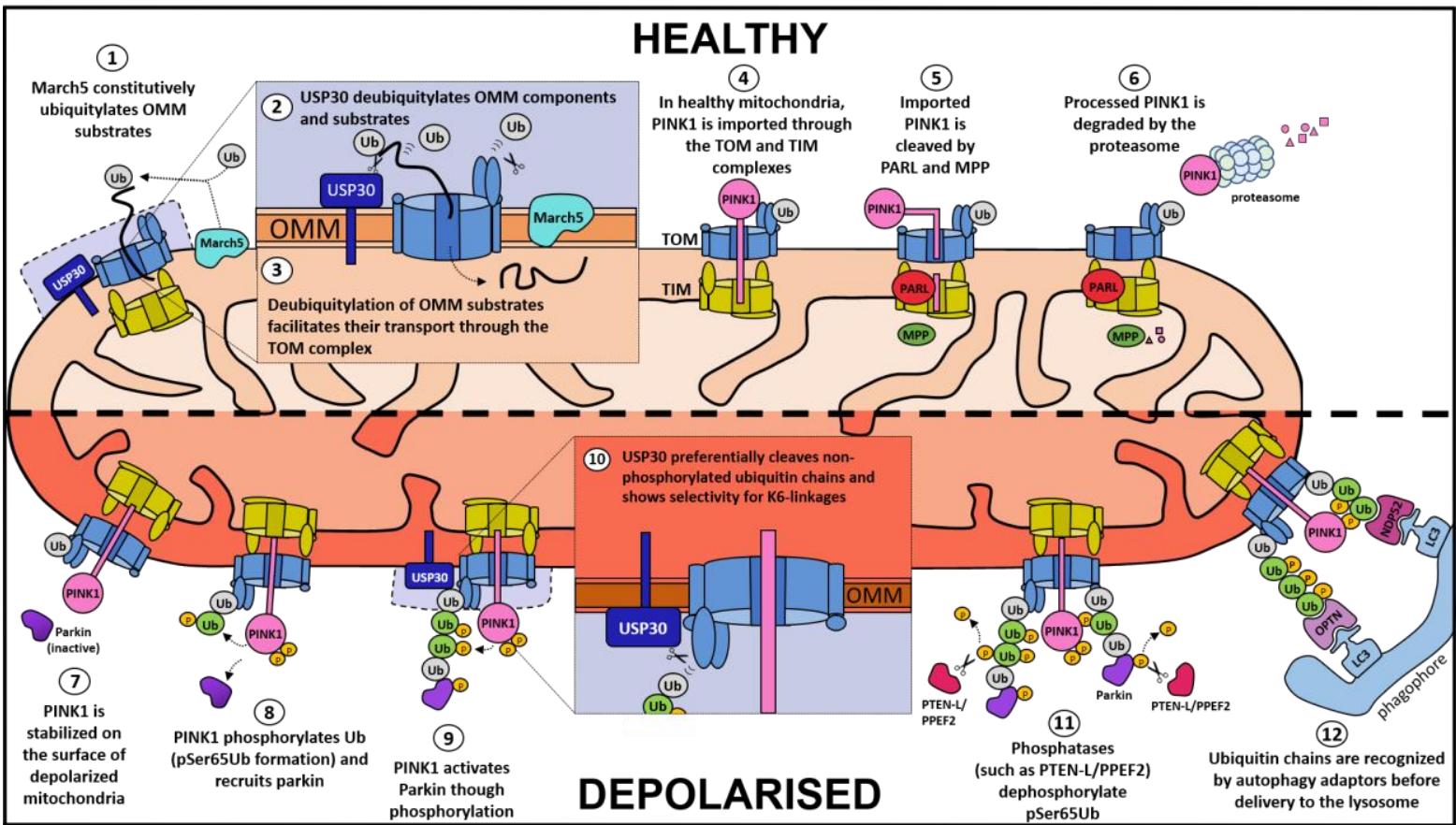

Figure 1. USP30 functions as a gatekeeper to activating the PINK1/parkin mitophagy pathway

1. E3 Ubiquitin (Ub) ligases, such as March5, ubiquitylate outer mitochondrial membrane (OMM) substrates which act as 'seeds' for PINK1/parkin-dependent mitophagy. 2. In spatially restricted regions, USP30 reduces ubiquitylation on OMM substrates under basal conditions. 3. USP30 deubiquitylates mitochondrial proteins, allowing their transport through the TOM complex (blue). 4. In healthy mitochondria and under basal conditions, PINK1 is imported through the TOM and TIM complexes (yellow). 5. While spanning the inner mitochondrial membrane (IMM), PINK1 is cleaved by PARL and MPP. 6. Processed PINK1 is then targeted to the proteasome for degradation. 7. When mitochondrial import is compromised, PINK1 becomes stabilised on the OMM. 8. PINK1 phosphorylates Ub (pSer65Ub) which marks OMM substrates. PINK1 activity also recruits parkin. 9. PINK1 also phosphorylates parkin, causing a conformational change resulting in increased parkin activity. 10. USP30 preferentially cleaves non-phosphorylated Ub chains and shows selectivity for K6-linkages. 11. PTEN-L and PPEF2 antagonize PINK1 activity by dephosphorylating parkin and Ub chains on the OMM. 12. Ub chains that remain on OMM substrates mediate mitophagy via their interactions with Ubbinding adapters allowing engulfment of the mitochondrion by the autophagosome and its subsequent degradation by the lysosome. 


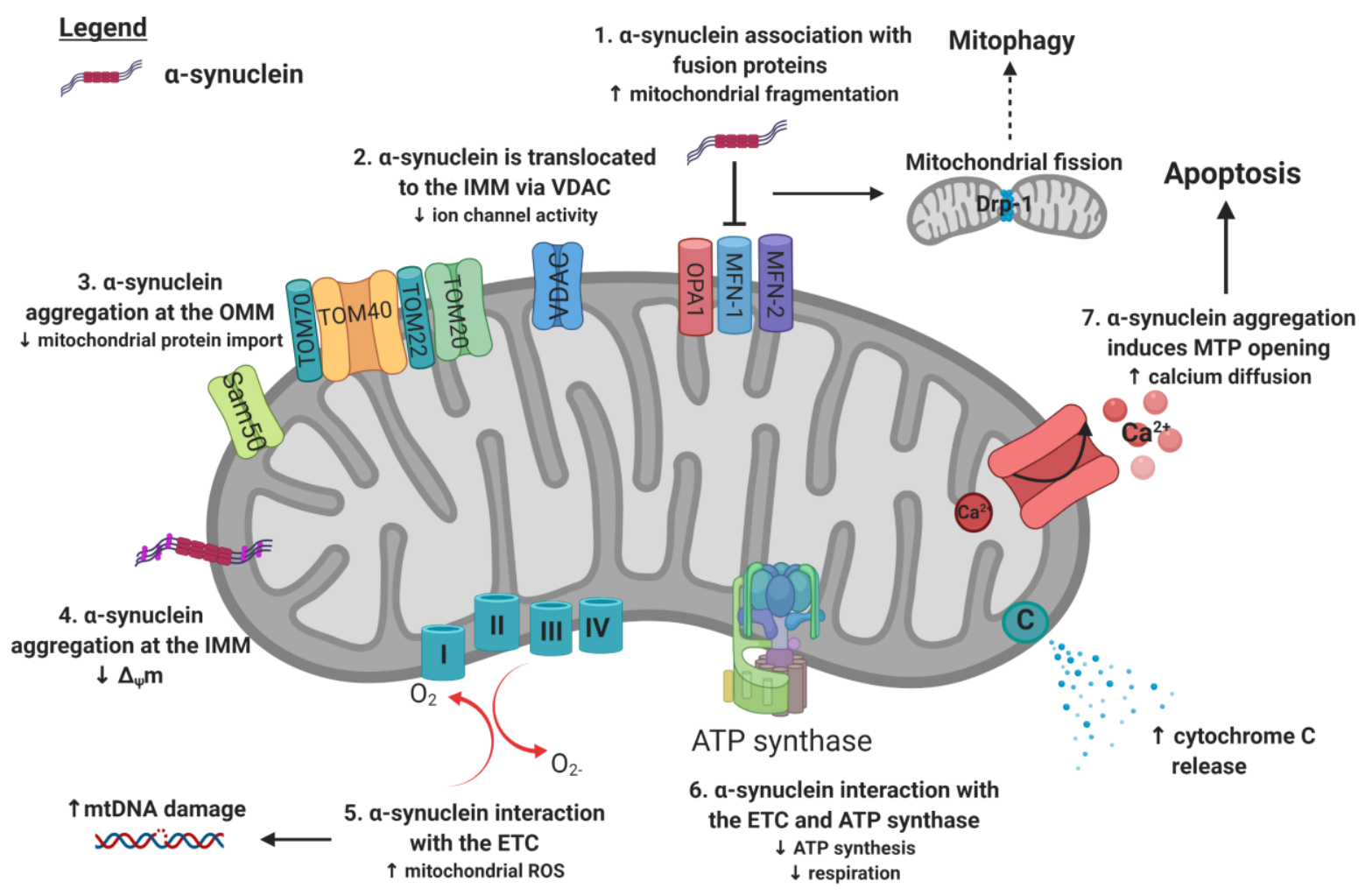

876 Figure 2. Aggregated and pathogenic forms of $\alpha$-synuclein preferentially bind to

877 mitochondria to affect mitochondrial function.

878 1) $\alpha$-synuclein interaction with fusion proteins such as OPA1, MFN-1, and MFN-2 has been 879 associated with increased Drp1-mediated fission and increased mitochondrial fragmentation.

880 2) $\alpha$-synuclein is translocated into the innter mitochondrial membrane (IMM) via VDAC and 881 blocks its ion channel activity. 3) $\alpha$-synuclein aggregation at different components of the 882 OMM, such as the TOM complex and the SAM (sorting and assembly machinery) complex, 883 impairs mitochondrial protein import [110]. 4) $\alpha$-synuclein aggregation at the intermembrnae 884 space (IMS) leads to mitochondrial membrane depolarization. 5) $\alpha$-synuclein interaction with 885 the electron transport chain (ETC), specifically at complexes $X$ and $Y$, increases the production 886 of mitochondrial reactive oxygen species (ROS), promoting mitochondrial DNA (mtDNA) 887 damage. 6) $\alpha$-synuclein interaction with components of the ETC results in decreased complex 888 I activity, decreased ATP production, and decreased respiratory capacity. 7) $\alpha$-synuclein 889 aggregation at the mitochondria induces MPTP opening, calcium diffusion, cytochrome $\mathrm{C}$ 890 release, and mitochondrial swelling, ultimately leading to apoptosis. 


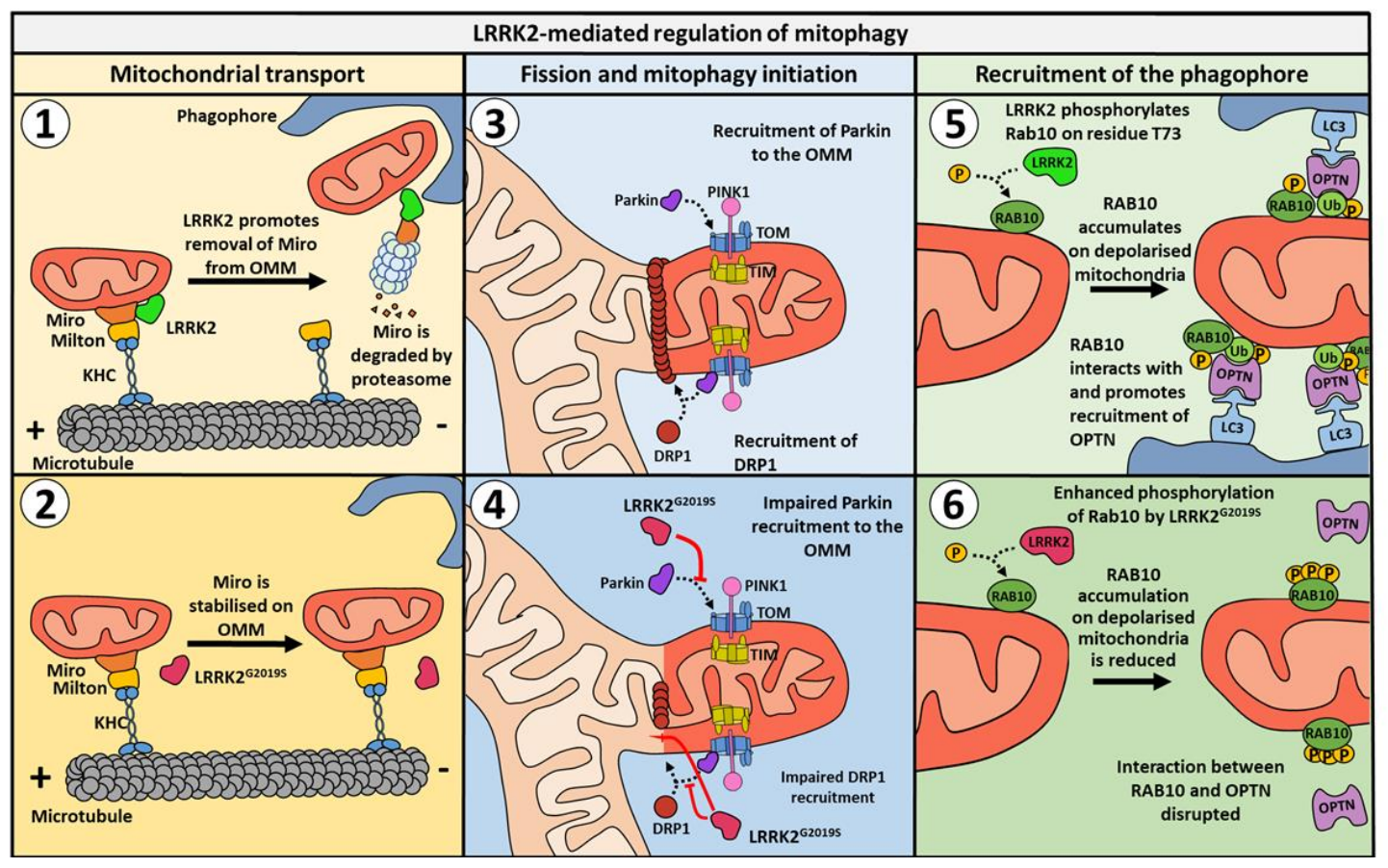

893 Figure 3. Normal and disease-associated functions of LRRK2 in regulating mitophagy.

894 1) LRRK2 can promote mitophagy by removing MIRO from the motor complex (including 895 Milton and kinesin (KHC)), thereby arresting mitochondrial transport and thus enabling 896 engulfment by autophagosomes. 2) Pathogenic G2019S substitution in LRRK2 disrupts this 897 function, thereby delaying the arrest of damaged mitochondria and slowing the initiation of 898 mitophagy. 3) Recruitment of parkin to the outer mitochondrial membrane (OMM) of 899 damaged mitochondria is one of the earliest stages of mitophagy. Co-ordinated recruitment 900 of Drp1 by PINK1 and parkin is essential for mitochondrial fission, which isolates damaged 901 mitochondria for subsequent degradation. 4) Increased LRRK2 kinase activity (overexpression 902 of LRRK2 ${ }^{\text {WT }}$ or LRRK2 ${ }^{\text {G2019S }}$ ) disrupts interactions between a) Parkin and TOM subunits, b) 903 Parkin and Drp1, and c) Drp1 and MiD51. This results in impaired PINK1/parkin-dependent 904 mitophagy. 5) LRRK2 phosphorylates RAB10 on residue T73 leading to its accumulation on 905 depolarized mitochondria. Here, it promotes recruitment and interacts with the ubiquitin906 binding adapter OPTN, thereby promoting mitophagy. 6) LRRK2 (G2019S and R1441C) impairs 907 later stages of PINK1/parkin-dependent mitophagy through increased phosphorylation of 908 RAB10, which inhibits its interaction with OPTN. This reduces the accumulation of RAB10 and 909 OPTN on depolarised mitochondria and attenuates mitophagy. 